\title{
Contents, Vol. 86, 1935
}

\section{Inhaltsverzeichnis.}

Eigenarbeiten.

Seite

Bartels, M., Unfall durch elektrischen Strom und Erkrankung des Zentralnervensystems I

Bergmeister, R., Über die Sichtbarkeitsgrenzen des optischen Spektrums 98

Blessig, E., Etwas über ,Lichtscheu”

Böck, J., Über einen Fall von hämatopoetischem Mark im heteroplastischen Knochen eines atrophischen Auges nebst

Bemerkungen zum Sektionsbefund bei Uveitis chronica 257 Boros, Bèla, Zur Frage der Ätiologie der Sehnervenentzündung

unbekannten Ursprungs 214

Gardilcic, A., Über Versuche mit einer neuartigen Trachom-

behandlung und deren Ergebnisse 121

Goebel, Carl, Zur Ätiologie und Therapie der sympathischen

Ophthalmie 204

Hesse, Erich, Freies Pigmentkörperchen in der Vorderkammer 26 Hinrichs, H., Einfluß von

Wetter und Jahreszeit auf die

Entstehung von Augenkrankheiten 269

Klein, M., Chorioidealsarkom bei diffuser Sarkomatose der

weichen Hirnhäute 287

- $\quad$ Nadel und Nadelhalter zur Vereinfachung der Mikro-

koagulationsoperation bei Netzhautablösung 127

Lewitsky, M., Über den Cysticercus intraocularis 300

Majoros, Joh., siehe Szalóczy, Karl.

Purtscher, Adolf, Beitrag zur Bildung freier Zysten in der

Vorderkammer 11

Rauh, Walter, Ausgedehnte Zerstörungen der Lider und der Gesichtshaut nach Lupus und Lues und ihre chirurgische Behandlung 193

RohrSchneider, W., Über den Frühjahrsgipfel der skrofulösen Augenerkrankungen 281

§afáŸ, Karl, Über eine Iriszyste im aphaken Auge, durch Elektrokoagulation geheilt 31

Sallmann, L., Netzhautfältelung bei raumbeengenden Prozessen in der Augenhöhle 18

- Die Trepanationszyklodialyse, eine Abänderung der Heineschen Zyklodialysenoperation Ill 
Saul, Hugo, Über das Schicksal der Patienten mit Keratitis parenchymatosa 199

Seefelder, R., Über familiäres Auftreten von Katarakt und

Poikilodermie (Poikilodermia vascularis atrophicans

Jacobi) 81

Szalóczy, Karl, und Joh. Majoros, Pantocain in der Augen-

heilkunde 220

- IV -

Seite

Verschiedenes.

Kunst und Auge. Von Dr. Oth. Huber-Glatus

37

Zu Dr. Pillats Vortrag: Über das Vorkommen von Chorio-iditis tbc. bei Lupus erythematodes.

Von DozentenDr. Rudolf Bergmeíster 224

Berichte über die ophthalmologische Literatur.

Ophthalmologische Therapie. I. Medikamentöse Therapie. VonDr. Eberhard Kunz--Betn. II.

Operative Therapie. III. Physi-kalische und Strahlentherapie. Von Prof. Wolfgang Hoffmann-

Königsberg 49

Bakteriologie, Parasitologie, Immunitätsforschung und Vergiftun-

gen. Von Doz. Dr. A. Pillat in Wien 132

Vegetative Physiologie und Pathologie. Von Priv.-Doz. Dr. W. Rauh

in Leipzig 157

Verletzungen - Versicherungswesen und soziale Medizin - Blinden-

wesen. Von Dr. R. Heßberg in Essen 167

Tränenorgane, Lider, Bindehaut. I. Tränenapparat. II. Lider.

III. Konjunktiva. IV. Trachom. Von Dr. Hugo Saul-Kiel 225

Kornea, Sklera, Episklera. Von Prof. Dr. H. Schmelzer in Erlangen 323

Gesellschaftsberichte.

Ophthalmolog. Gesellschaft in Wien. Sitzung vom 21. Januar 193566

Sitzungen vom 18. März und

1. April 1935237 Sitzung vom 21. Mai 1935333

39. Tagung der Vereinigung mitteldeutscher Augenärzte zu Leipzigam 8. und 9. Dezember 1934.

Nach Eigenberichten zusammen-gestellt von Dozenten Dr. K. Velhagen jun., Halle 242

Verein Rheinisch-Westfälischer Augenärzte. Sitzung vom 18. November 1934 m Düsseldorf 340

Bericht über die 26. wissenschaftliche Sitzung der augenärztlichenVereinigung des

Oberbergamtsbezirks Dortmund. Sitzungvom 16. Februar 1935 in Dortmund 343

Gesellschaft der Augenärzte von Zurich. Sitzung vom i.März 1935187

Deutsche Ophthalmologische Gesellschaft in der Tschechoslowaki-

schen Republik. Sitzung vom 23. und 24. Februar 1935 . 347

Diagnose und Therapie $\quad 76,190,251,358$

Nachtrag zu: Zur Vorgeschichte der Wiener Àagenkliniken. Von

Doz. Dr. I. Fischer in Wien (Bd. 85, 5/6) 80

Buch $1 / 8$ esprechung 255

Personalien und Tagesnachrichten $\quad 80,192,256,362$

S. Sachregister zu Band $86 \quad 363$

Namenregister zu Band $86 \quad 368$ 
